\title{
The fate of incoming stimuli during NREM sleep is determined by spindles and the phase of the slow oscillation
}

\begin{abstract}
Manuel Schabus ${ }^{1,2}{ }^{*}$, Thien Thanh Dang-Vu ${ }^{1}$, Dominik Philip Johannes Heib ${ }^{2}$, Mélanie Boly ${ }^{1}$, Martin Desseilles ${ }^{1}$, Gilles Vandewalle ${ }^{1}$, Christina Schmidt ${ }^{1}$, Geneviève Albouy ${ }^{1}$, Annabelle Darsaud ${ }^{1}$, Steffen Gais ${ }^{1}$, Christian Degueldre ${ }^{1}$, Evelyne Balteau ${ }^{1}$, Christophe Phillips ${ }^{1}$, André Luxen ${ }^{1}$ and Pierre Maquet ${ }^{1}$ *
\end{abstract}

${ }^{1}$ Cyclotron Research Centre, University of Liège, Liège, Belgium

${ }^{2}$ Laboratory for Sleep and Consciousness Research, University of Salzburg, Salzburg, Austria

\section{Edited by:}

Michael Czisch, Max Planck Institute of Psychiatry, Germany

Reviewed by:

Renate Wehrle, University Clinic Regensburg, Germany

Kátia C. Andrade, Brain

Institute/Federal University of Rio

Grande do Norte, Brazil

*Correspondence:

Manuel Schabus, Department of Psychology, University of Salzburg, Hellbrunnerstr.34, 5020 Salzburg, Austria.

e-mail: manuel.schabus@sbg.ac.at Pierre Maquet, Cyclotron Research Centre (B30), University of Liège, Sart Tilman, 4000 Liège, Belgium. e-mail:pmaquet@ulg.ac.be
The present study aimed at identifying the neurophysiological responses associated with auditory stimulation during non-rapid eye movement (NREM) sleep using simultaneous electroencephalography (EEG)/functional magnetic resonance imaging (fMRI) recordings. It was reported earlier that auditory stimuli produce bilateral activation in auditory cortex, thalamus, and caudate during both wakefulness and NREM sleep. However, due to the spontaneous membrane potential fluctuations cortical responses may be highly variable during NREM. Here we now examine the modulation of cerebral responses to tones depending on the presence or absence of sleep spindles and the phase of the slow oscillation. Thirteen healthy young subjects were scanned successfully during stage 2-4 NREM sleep in the first half of the night in a $3 T$ scanner. Subjects were not sleep-deprived and sounds were post hoc classified according to (i) the presence of sleep spindles or (ii) the phase of the slow oscillation during $( \pm 300 \mathrm{~ms})$ tone delivery. These detected sounds were then entered as regressors of interest in $\mathrm{fMRI}$ analyses. Interestingly wake-like responses although somewhat altered in size and location - persisted during NREM sleep, except during present spindles (as previously published in Dang-Vu et al., 2011) and the negative going phase of the slow oscillation during which responses became less consistent or even absent. While the phase of the slow oscillation did not alter brain responses in primary sensory cortex, it did modulate responses at higher cortical levels. In addition EEG analyses show a distinct N550 response to tones during the presence of light sleep spindles and suggest that in deep NREM sleep the brain is more responsive during the positive going slope of the slow oscillation. The presence of short temporal windows during which the brain is open to external stimuli is consistent with the fact that even during deep sleep meaningful events can be detected. Altogether, our results emphasize the notion that spontaneous fluctuations of brain activity profoundly modify brain responses to external information across all behavioral states, including deep NREM sleep.

Keywords: AEP, sleep, spindles, spontaneous activity, slow-wave phase, EEG/fMRI, fMRI, EEG

\section{INTRODUCTION}

Classically, brain processes are considered as essentially reflexive and mainly driven by external stimuli. In this perspective, brain function is predominantly geared interpreting incoming stimuli and programming motor output. Another view posits that the bulk of brain activity is intrinsic, spontaneous (i.e., it emerges in the absence of any identified external stimulus), and continuously maintains and processes information (Raichle, 2006). Consistent with this view, the energy required for the brain to respond to external stimuli is extremely small compared to the ongoing amount of energy that the brain normally and continuously expends (Raichle and Mintun, 2006). During wakefulness, spontaneous fluctuations of brain activity profoundly modify brain responses to external information. For instance, conscious perception of the external world is dependent upon pre-stimulus activity in the somatosensory (Palva et al., 2005; Boly et al., 2007) as well as visual domain (Hanslmayr et al., 2007; Hesselmann et al., 2008a,b). Likewise, cortical responses to external stimuli should be modulated by the spontaneous non-rapid eye movement (NREM) sleep background activity materialized by sleep spindles and slow-waves (SW), which are associated with specific activity patterns in thalamic and cortical neurons during sleep. Accordingly, animal (Steriade, 1991) and human (Elton et al., 1997; Cote et al., 2000) studies clearly suggested that sensory transmission is blocked at the thalamic level during sleep spindles due to the recurrent inhibition of thalamocortical neurons by reticular thalamic cells and thereby might even herald individual resilience to disruptive stimuli such as environmental noise in human subjects (Dang-Vu et al., 2010). Likewise, 
processing of somatosensory inputs during deep NREM sleep was shown to be strongly influenced by the phase of the slow oscillation at which stimuli were delivered (Massimini et al., 2003). The amplitude of early evoked potentials increased during the downswing of the electroencephalography (EEG) slow fluctuation, potentially in relation to a progressive decrease in input resistance of cortical neurons (Contreras et al., 1996) and heightened probability of synaptic release (Massimini and Amzica, 2001) which is highest during the transition from the cellular down to up state (i.e., negative-to-positive EEG slope transition; Massimini et al., 2003).

In humans, EEG (Bastuji and Garcia-Larrea, 1999) and neuroimaging (Portas et al., 2000) studies primarily insisted on the persistence of brain responses to auditory stimuli. With regards to EEG the extent of residual auditory information processing during sleep has been extensively studied using event-related potentials (ERPs; for review see Campbell and Colrain, 2002). Therein it has been consistently reported that the early N1 component is gradually decreased from sleep onset to stage 2 NREM sleep whereas the P2 amplitude is increased (Campbell et al., 1992). Latter effect has also been associated with an attenuation of the classical processing negativity (de Lugt et al., 1996) which is thought to reflect additional attentional processing of attenuated stimuli during waking (Näätänen et al., 1992). Consequently, absence of this overlapping negativity is regarded to reflect inhibition of information processing (Campbell et al., 1992). In addition, during the occurrence of sleep spindles the amplitude of the P2 component has been found to be even more amplified (Elton et al., 1997). According to the authors, this reflects a further inhibition of stimulus processing during sleep. Besides these modified waking components NREM sleep (auditory) ERPs further consist of unusual large amplitude components. Specifically these are the N350 (between 250 and $400 \mathrm{~ms}$ ), the N550 (between 500 and $800 \mathrm{~ms}$ ), and the P900 (between 800 and $1300 \mathrm{~ms}$ ). While the N350 possibly reflects an active inhibition of sensory processing during sleep onset, the later components (N550 and P900) form part of the very large amplitude stimulus-elicited K-complex and thus might reflect or overlap with the generation of delta oscillations for the sake of sleep protection (for review see Bastien et al., 2002; Colrain and Campbell, 2007).

Neuroimaging studies suggested that the brain can even detect meaningful auditory events (like a subjects own name) during NREM sleep in which it elicits significant responses in the amygdala and prefrontal cortex in addition to the bilateral activation of auditory cortex, thalamus, and caudate nuclei seen in response to simple auditory stimuli (Portas et al., 2000). In contrast, Czisch et al. (2002) reported that the brain response to auditory stimulation was decreased during NREM sleep as compared to wakefulness, interpreting their results in terms of a sleep-protective deactivation of primary sensory areas. In a follow up study the same authors demonstrated that the stimulus-induced negative BOLD effects - again primarily found in light NREM sleep correlated positively with EEG signs of hyperpolarization (i.e., K-complexes and delta power) suggesting "true cortical deactivation upon stimulus presentation"(Czisch et al., 2004). However, all these studies considered NREM sleep as a homogeneous and steady state and did not account for the potential influence of spontaneous ongoing brain activity. The data reported in this article precisely aimed at characterizing sound processing during specific NREM sleep features using simultaneous EEG/functional magnetic resonance imaging (fMRI) recordings. Also note that previous fMRI studies used continuous stimulation (up to $42 \mathrm{~s}$ ) and probably measured to a considerable part induced changes in brain states (such as arousals or increased gating) whereas the current paper makes use of the more advanced event-related paradigm focusing directly on evoked brain activity.

Here we now use simultaneous EEG and fMRI in order to characterize brain responses to tones during light and deep NREM sleep in non sleep-deprived healthy individuals. Specifically, we examined brain responses to auditory stimuli during NREM stage 2-4 sleep in relation to the presence of sleep spindles (fMRI data published previously in Dang-Vu et al., 2011) and the phase of the slow oscillation.

\section{MATERIALS AND METHODS}

Thirteen - out of 19 recorded ( 9 females; age range $=18-25$; mean age $=21.3$ ) -healthy subjects were successfully scanned during the first half of the night in a Siemens Allegra $3 \mathrm{~T}$ scanner with a full 64 channel montage of MR-compatible EEG electrodes.

Participants were right-handed and gave their written informed consent. After the experiment participants received a financial compensation for their participation in this study, which was approved by the Ethics Committee of the Faculty of Medicine of the University of Liège. Participants were free of any medical, traumatic, psychiatric, or sleep disorder history, as assessed by a semi-structured interview. No participant complained of excessive daytime sleepiness (Epworth Sleepiness Scale; Johns, 1991) or sleep disturbances (Pittsburgh Sleep Quality Index Questionnaire; Buysse et al., 1989). All participants had normal scores at the Beck Anxiety and Beck Depression Inventory, and were non-smokers, moderate caffeine and alcohol consumers, as well as refraining from medication. None had worked on night shifts during the last year or traveled through more than one time zone during the last 2 months. Extreme morning and evening types, as assessed by the Horne-Ostberg Questionnaire (Horne and Ostberg, 1976), were not included.

Subjects were not sleep-deprived and followed a 4-day constant sleep schedule, as controlled by wrist actigraphy (Actiwatch, Cambridge Neuroscience, UK) and sleep diaries. Volunteers were requested to refrain from all caffeine and alcohol-containing beverages and intense physical activity for 3 days before participating in the study. Subjects reported to the laboratory at $9 \mathrm{pm}$.

\section{EEG AND fMRI METHODOLOGY}

Electroencephalography was recorded simultaneously to fMRI acquisitions, during the first half of the night, utilizing two MRcompatible 32-channel amplifiers (BrainAmp MR plus, Brain Products $\mathrm{GmbH}$, Gilching, Germany) and a MR-compatible EEG cap (BrainCap MR, Falk Minow Services, HerrschingBreitbrunn, Germany) with 64 ring-type electrodes. EEG caps included 62 scalp electrodes which were online referenced to $\mathrm{FCz}$, as well as 1 electrooculogram (EOG) and 1 electrocardiogram (ECG) channel. Using abrasive electrode paste (ABRALYT 2000; 
FMS, Herrschings-Breitbrunn, Germany) electrode-skin impedance was kept below $5 \mathrm{k} \Omega$ (in addition to the $5-\mathrm{k} \Omega$ resistor built into the electrodes). To avoid movement-related EEG artifacts, the subjects' head was immobilized in the head-coil by a vacuum pad.

Electroencephalography was digitized at $5000 \mathrm{~Hz}$ sampling rate with a $500-\mathrm{nV}$ resolution. Data were analog-filtered by a band limiter low-pass filter at $250 \mathrm{~Hz}(30 \mathrm{~dB} /$ Octave) and a high-pass filter with $10 \mathrm{~s}$ time constant corresponding to a high-pass frequency of $0.0159 \mathrm{~Hz}$. Data were transferred outside the scanner room through fiber optic cables to a personal computer where the EEG system running Vision Recorder Software v1.03 (Brain Products GmbH, Gilching, Germany) was synchronized to the scanner clock. Sleep EEG was monitored online with Brain Products RecView Software.

For analysis, EEG data were low-pass filtered (FIR filter, $-36 \mathrm{~dB}$ at $70 \mathrm{~Hz}$ ), down-sampled to $250 \mathrm{~Hz}$, and re-referenced to the mastoids. Scanner gradient artifacts were removed in Vision Analyzer, using an adaptive average subtraction (Allen et al., 2000). Ballistocardiographic (BCG) artifacts were removed using an algorithm based on independent component analysis (Srivastava et al., 2005). Sleep staging followed standard criteria (Rechtschaffen and Kales, 1968), and identified periods of stage 2-3 or stage 3-4 NREM sleep, free of any artifact, during which the EEG and fMRI data were analyzed. Only stable stage $2-3$ or stage 3-4 epochs lasting more than $2 \mathrm{~min}$ were considered for the analysis of sleep spindles (for details see Dang-Vu et al., 2011) and SW activity, respectively. For EEG spindle and SW analysis we used Fz, Cz, and Pz. For display and statistics we selected the $\mathrm{Cz}$ lead as this electrode allows to depict spindle as well as SW effects.

Functional MRI time series were acquired using a $3 \mathrm{~T}$ MR scanner (Allegra, Siemens, Germany). Multislice T2*weighted fMRI images were obtained with a gradient echoplanar sequence using axial slice orientation (32 slices; voxel size: $3.4 \mathrm{~mm} \times 3.4 \mathrm{~mm} \times 3 \mathrm{~mm}$; matrix size $=64 \times 64 \times 32$; $\mathrm{TR}=2460 \mathrm{~ms} ; \mathrm{TE}=40 \mathrm{~ms}$; flip angle $=90^{\circ}$; delay $=0$ ). Subjects were scanned during the first half of the night, starting at around midnight. They were asked to relax, try to sleep in the scanner and not pay attention to occasional tones, while fMRI and EEG data were acquired continuously. They stayed until they indicated by button press that they would like to go out, or for a maximum of 4000 volumes (about $164 \mathrm{~min}$ ). The number of acquired volumes varied between 1195 and 4000 [3401 \pm 965 volumes or $139 \pm 40 \mathrm{~min}($ mean $\pm \mathrm{SD})]$. A structural T1weighed 3D MP-RAGE sequence $(\mathrm{TR}=1960 \mathrm{~ms}, \mathrm{TE}=4.43 \mathrm{~ms}$, inversion time $=1100 \mathrm{~ms}, \quad F O V=230 \mathrm{~mm} \times 173 \mathrm{~mm}$, matrix size $=256 \times 192 \times 176$, voxel size $=0.9 \mathrm{~mm} \times 0.9 \mathrm{~mm} \times 0.9 \mathrm{~mm})$ was also acquired in all subjects.

In addition, waking sessions before and after sleep were selected to identify default brain activations associated with the occurrence of tones during wakefulness (TW). The analysis of waking fMRI data characterized the brain responses to TW compared to waking baseline activity.

Functional volumes were analyzed by using Statistical Parametric Mapping 8 (SPM8; http://www.fil.ion.ucl.ac.uk/spm/) implemented in MATLAB (MathWorks). The series of consecutive fMRI volumes corresponding to selected stage $2-3$, stage $3-4$, or wake periods were selected from the complete fMRI time series and constituted a "session." fMRI time series were corrected for head motion, spatially normalized (voxel size $=2 \mathrm{~mm} \times 2 \mathrm{~mm} \times 2 \mathrm{~mm}$; resampled using spline interpolation) to an echo-planar imaging template conforming to the Montreal Neurological Institute space, and spatially smoothed with a Gaussian kernel of 8-mm FWHM.

The analysis of fMRI data, based on a mixed effects model, was then conducted in two serial steps, accounting respectively for intraindividual (fixed) and interindividual (random effects) variance in SPM8.

In a first analysis (as previously published in Dang-Vu et al., 2011), spindles were identified on band pass filtered EEG data between 11 and $15 \mathrm{~Hz}$, using an automatic detection algorithm by thresholding the spindle root mean square signal at its 95th percentile and post hoc visually checking for correct classification (Mölle et al., 2002). Brain responses related to spontaneously occurring spindles and SW have already been described elsewhere (Schabus et al., 2007; Dang-Vu et al., 2008) and are not reported in the following. Responses to sounds corresponded to systematic deviations of BOLD signal over and above the baseline activity during NREM sleep having taken into account the activity related to characteristic oscillations of NREM sleep.

In a second analysis, two tone categories were considered, depending on whether the stimulus appeared before [tone 0 $300 \mathrm{~ms}$ pre-SW-peak (TPre)] or (max. $300 \mathrm{~ms}$ ) after the peak negativity of the (stage 3-4) slow oscillation [post-SW-peak (TPost)]. The SW-peak negativity was defined as the highest negative component (a negative peak between two zero crossings with voltage $<-35 \mu \mathrm{V})$ in a frontal EEG array (Fp1, Fp2, F3, F4, F7, F8, Fz, F1, F2, AF3, AF4, F5, F6, AF7, AF8, AFz). We assessed brain responses as simple main effects of tones relative to baseline activity (independent of the phase of the slow oscillation) as well as differential main effects between the two tone categories (pre to post SW-peak differences). In order to find the instant at which the sound volume changed at its fastest rate, we identified the downward zero crossing of the second derivative of the Gaussian sound envelope. This timepoint ( $88 \mathrm{~ms}$ after sound onset) was taken as the moment at which the sound became detectable by the volunteer which we consequently used as onset for EEG and AMRI analysis.

In order to take into account the effects of all identifiable neural events on the BOLD signal during sleep, sleep spindles and SW power were also modeled in the analysis. To take into account artifacts related to cardiac cycle, an estimation of R-R intervals derived from ECG was included as regressor of no interest in all individual design matrices. Movement parameters estimated during realignment (translations in $x, y$, and $z$ directions and rotations around $x, y$, and $z$ axes) and a constant vector were also included in the matrix as variables of no interest. High-pass filtering was implemented in the matrix design using a cut-off period of $128 \mathrm{~s}$ to remove low frequency drifts from the time series. Serial correlations in fMRI signal were estimated using an autoregressive (order 1) plus white noise model and a restricted maximum likelihood (ReML) algorithm.

Statistical inferences were conducted after correction for multiple comparisons either on the whole brain volume $\left(p_{\mathrm{FWE}}<0.05\right)$ or for regions of interest previously identified in the literature using small volume correction ( $\left.p_{\mathrm{SVC}}<0.05\right)$. The Supplementary Material (Table S1) is not corrected for multiple comparisons, and 
consequently no statistical inferences were conducted for those areas.

\section{AUDITORY STIMULATION DURING SLEEP}

Throughout sleep pure tones were presented binaurally using headphones. Tones had a frequency of $400 \mathrm{~Hz}$, a duration of $300 \mathrm{~ms}$ and were presented at each TR $(2460 \mathrm{~ms})$ with a probability of $70 \%$. Within a given volume the sound could occur anywhere with the $2460 \mathrm{~ms}$ scan frame. Yet in $30 \%$ of the cases the sound was not presented within that volume. This resulted in a median ISI of $2910 \mathrm{~ms}$ and SD of $10706 \mathrm{~ms}$.

The intensity of tones was held constant throughout the night and adjusted individually during a test scanning session reproducing the same background noise than the experimental one. Subjects were requested to adjust the tone loudness to a level which was discernible but not disturbing. For definition of the early auditory ERP components we used latency ranges similar to Crowley and Colrain (2004) but shifted them $40 \mathrm{~ms}$ in time (N1: 115-190 and P2: 190-290 ms) after stimulus onset in order to account for delayed responses in fMRI scanner environments (cf. Novitski et al., 2001).

TPre and TPost epochs were classified on $0.5-20 \mathrm{~Hz}$ bandpass filtered EEG data if a frontal SW peak "exceeding" $-35 \mu \mathrm{V}$ was identified within $300 \mathrm{~ms}$ after or before the tone, respectively (cf. Figure 1). Each analyzed epoch then ranged from $-1500 \mathrm{~ms}$ pre to $1500 \mathrm{~ms}$ post tone stimulation. One subject had to be excluded for SW-EEG averaging due to bad signal quality. Statistical analysis between TPre and TPost were conducted on SW phase-sorted averages (i.e., after identifying all TPre and TPost SW-peaks, we realigned the single trials to the SW-peak, and then averaged across

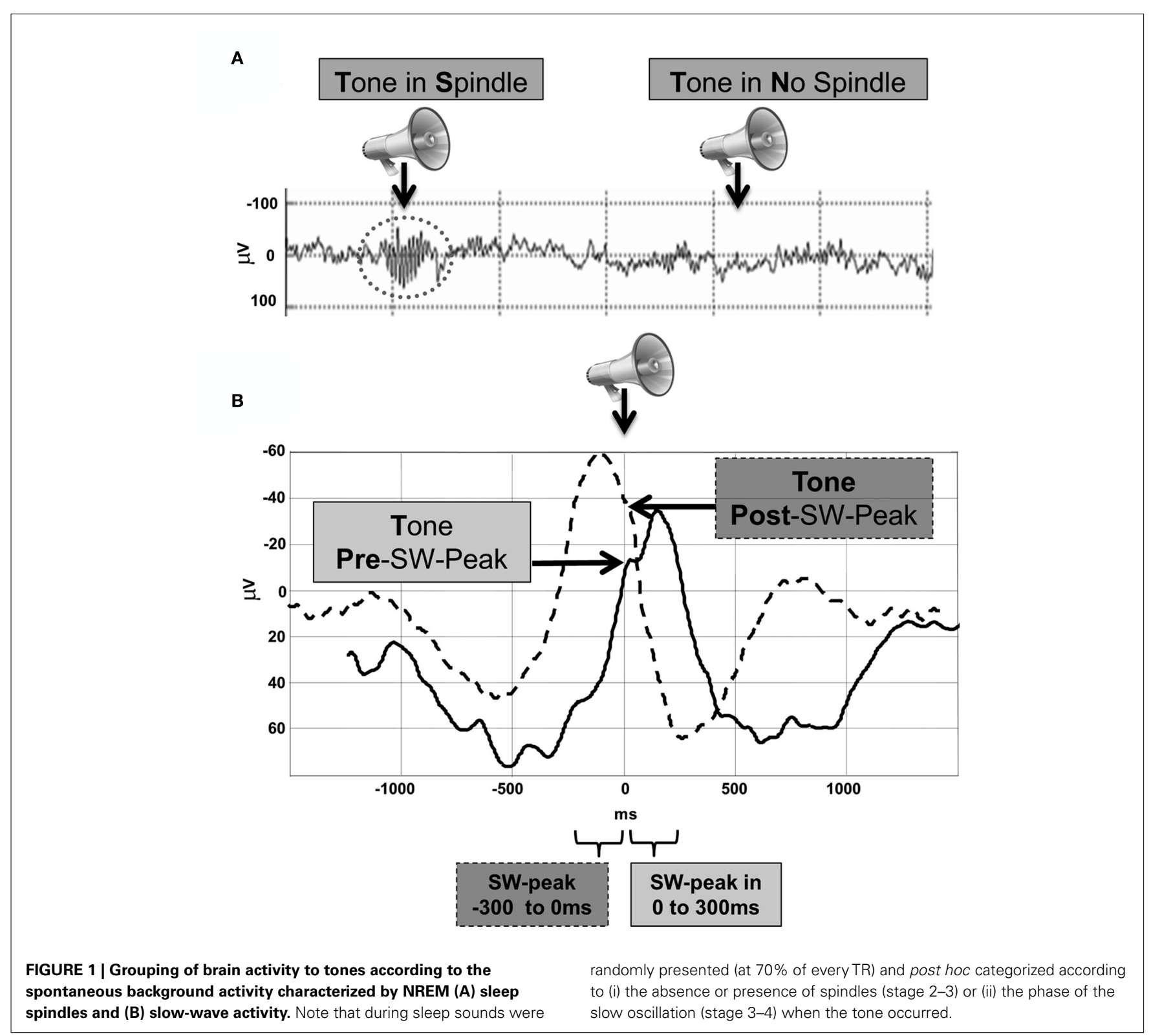


trials and subjects per tone category) which were previously lowpassed filtered at $4 \mathrm{~Hz}$. In order to be able to interpret the effect of tones on the slow oscillation we also calculated spontaneous SW's (in a time window of -1500 to $0 \mathrm{~ms}$ before tone stimulation) for comparison. The peak negativity ("exceeding" $-35 \mu \mathrm{V}$ ) of these spontaneous SW's had to occur $400 \mathrm{~ms}$ after epoch start $(-1100 \mathrm{~ms}$ pre-stimulus) as well as at least $800 \mathrm{~ms}$ before tone onset in order to only identify spontaneous SW's without any influence of surrounding tones on the spontaneous waveform. In addition, we classified SWs depending on the amplitude of the peak negativity (small SW: $\max .-50 \mathrm{mV}$; big SW: more negative than $-50 \mathrm{mV}$ ) for further analyses. For stage 2-3 sleep analysis of spindles, tones were categorized according to their occurrence outside (TN) or within detected spindles (TS; cf. Figure 1, Dang-Vu et al., 2011).

\section{BOLD SIGNAL MODULATION BY SPINDLES (fMRI DATA REPRODUCED FROM DANG-VU ET AL., 2011)}

The mean number of sounds delivered without (TN) or with ongoing spontaneous spindles (TS) per subject was 534.3 (SD = 198.8) and $30(\mathrm{SD}=11.2)$, respectively. As reported previously (Dang$\mathrm{Vu}$ et al., 2011), tones delivered in the absence of sleep spindles (TN) were also - like TW (Figure 2A) - associated with responses in thalamus and primary auditory cortex (Heschl's transverse gyrus) (Figure 2B), confirming that sounds can be processed in stage 2-3 NREM sleep. Significant additional TN responses were found in a set of cortical and subcortical areas, in the pons, cerebellum, middle frontal gyrus, precuneus, and posterior cingulate gyrus, all areas known to respond to auditory stimuli (Holcomb et al., 1998; Portas et al., 2000; Gaab et al., 2003). In contrast, at the same statistical threshold ( $p<0.05$

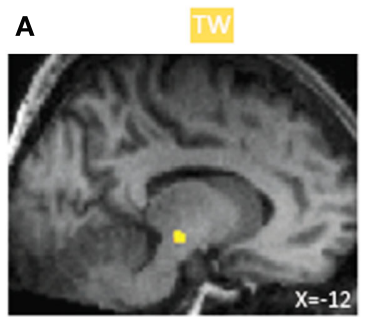

B
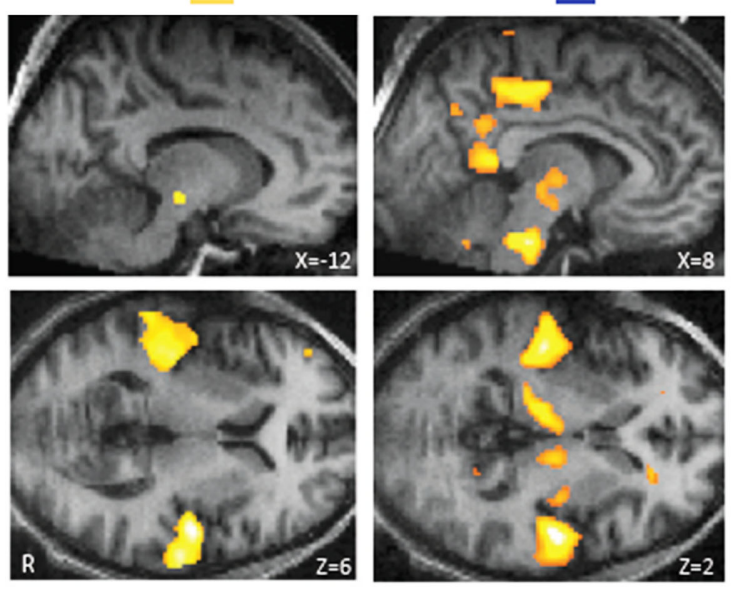

C
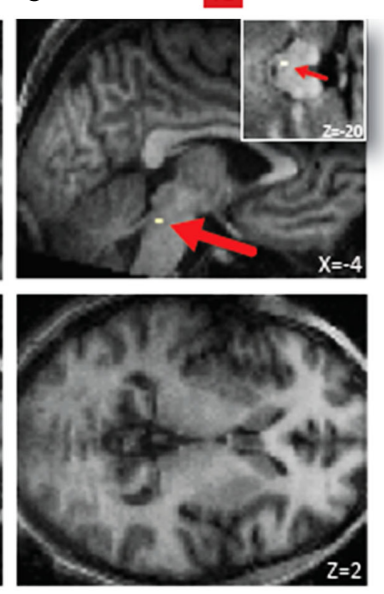

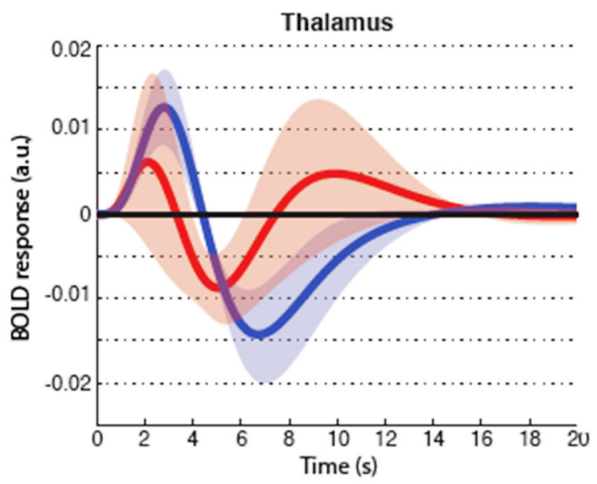

FIGURE 2 | Brain regions activated in relation to tones during waking, light NREM sleep but outside sleep spindles and light sleep during spindles (data reproduced from Dang-Vu et al., 2011). (A) Significant responses associated with tones presented during waking (TW). Note that tones during wakefulness (TW) induced responses in the primary auditory cortex (Heschl's transverse gyrus) and the thalamus, in an area compatible with the medial geniculate nucleus. (B) Significant responses associated with tones presented during stage 2-3 sleep, in the absence of ongoing spindles (TN). These responses are located in the thalamus, primary auditory cortex, brainstem, cerebellum, middle frontal gyrus, precuneus, and posterior cingulate gyrus. The brainstem response encompasses areas compatible with

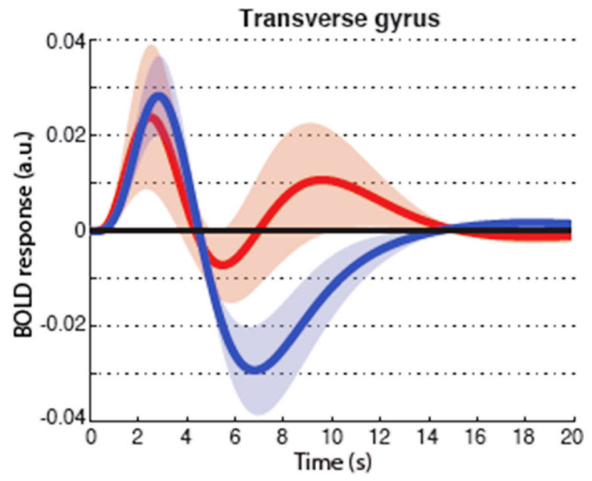

the cochlear nuclear groups the trapezoid bodies and the superior olivary complex. (C) Significant responses associated with tones presented within spindles in stage 2-3 sleep (TS). Here neural populations that process sound are found in the nuclei of the lateral lemniscus of the brainstem (insert, marked by arrow). Lower panels depict the fitted responses in the thalamus $[x=-12, y=-22, z=-6$; left panel $]$ and the auditory cortex $[x=58$, $y=-14, z=6$; right panel] associated with sounds delivered with (red) or without (blue) ongoing spontaneous spindles. The curves correspond to the mean and the shaded areas to the SEM. Functional results are displayed on an individual structural image (displayed at $p<0.001$, uncorrected). (Modified from Dang-Vu et al., 2011. Copyright by the National Academy of Sciences.) 
corrected), neither significant response to TS was found in the thalamus nor in the Heschl's gyri, but rather in a small area in the caudal aspect of the inferior colliculus (Figure 2C). Furthermore, we characterized the regional brain responses according to whether or not $\mathrm{TN}$ was followed by an induced K-complex and found that tones elicited larger responses in bilateral primary auditory cortex and ventral prefrontal cortex in the presence of an induced K-complex (for details refer to Dang-Vu et al., 2011).

\section{RESULTS}

The mean number of TW per subject was 161.3 (SD =96.5). Last but not least, the total number of tones during deep (stage 34) sleep was on average $524.5(\mathrm{SD}=166.5)$ per subject. Out of these the mean number of TPre and TPost SW-peak was 142.8 $(\mathrm{SD}=43.8)$ and $153.2(\mathrm{SD}=42.4)$ per subject respectively.

\section{BOLD SIGNAL MODULATION BY PHASE OF THE SLOW OSCILLATION}

Functional MRI results are summarized in Table $\mathbf{1}$ and being illustrated in Figure 3.

Data reveal that the thalamus continues to respond to tones during deep NREM sleep, when considering all stimuli irrespective of their phase relation with the slow oscillation. No thalamic difference in response is detected between the two categories of sounds (TPre and TPost SW-peak). Yet, thalamic responses to tones during deep sleep appeared spatially more extended than during waking (cf. Figure 3A). This finding remains qualitative as direct comparison between waking and sleep responses is precluded by the difference in baseline.

Likewise, primary auditory cortex (Heschl Gyrus) responds to tones during deep NREM sleep irrespective of their phase relation with the slow oscillation and there is no significant difference in responses to tones presented before (TPre) or after (TPost) the SW-peak negativity. By contrast, the superior temporal gyrus (STG), a higher auditory association cortex, responded more during the positive going slope of the slow oscillation (TPost) than during the negative going slope (TPpre; for additional areas also see Table S1 in Supplementary Material). This finding confirms that the brain response during sleep depends upon the phase of the slow oscillation characterizing deep NREM sleep.

\section{EEG RESPONSES TO TONES DURING SLEEP}

In addition to the fMRI responses we also focused on the corresponding EEG changes during the presentation of sounds in light and deep NREM sleep (Figures 4 and 5).

Analogous to the data presented above we compared delivered tones during the presence or absence of stage 2-3 sleep spindles (cf. Figure 4B) as well as the dependence of the evoked response with regards to the spontaneously present phase of the slow oscillation during sound delivery (cf. Figure 5).

Statistical analysis revealed that EEG responses to tones presented during (TS) or outside spontaneously occurring stage 2-3 sleep spindles (TN) differed markedly at around $650 \mathrm{~ms}$ $\left(t_{11}=3.76, p=0.001\right)$. Tones occurring during the presence of a sleep spindle evoked a late negativity (N550; Figure 4B) which is also considerably larger than that in response to tones during either waking, stage 2-3 or stage 3-4 sleep in general (cf. Figure 4A).

In addition for deep NREM sleep, it is evident that the evoked response to tones across stage 3-4 sleep (cf. Figure 5A) is markedly different from either tones occurring before or after SW-peaknegativities. Importantly, tones after SW-peak negativity (TPost) evoke a distinctly stronger positive synchronization than tones arriving pre-SW-peak (cf. Figures 5B,C).

Statistical analysis reveals that whereas the peak negativity of the slow oscillation is not altered if tones are presented before as compared to post SW-peak negativity $\left(t_{11}=0.84, p=0.42\right)$, robust effects of SW phase are revealed when focusing on differences during the late positive wave (Figure 5B, orange shading). Here, the positive component appears enhanced (150-550 ms post peak negativity) if the tone was arriving at the positive going slope (TPost) of the slow oscillation (peak amplitude: $t_{11}=5.55$, $p<0.001$; area under positive component: $t_{11}=5.65, p<0.001$; cf. Figure 5C). Before tone presentation the positive components of TPre and TPost are not significantly different from each other (peak amplitude: $t_{11}=2.06, p=0.064$; area before SW-peak: $\left.t_{11}=-0.043, p=0.97\right)$. Note that for valid statistical comparison we used phase-sorted averages. As there is no influence of time jitter (300 ms grouping windows for TPre and TPost) on averaging in that case (Figure 5C) the component amplitudes are enhanced (as compared to Figure 5B) and better reflect real single trial data.

Table 1 | Brain responses to tones during deep NREM sleep.

\begin{tabular}{|c|c|c|c|c|c|c|c|c|c|c|c|c|}
\hline \multirow[t]{2}{*}{ Region } & \multicolumn{6}{|c|}{ SW phase independent effect } & \multicolumn{6}{|c|}{ SW phase dependent differences (TPost > TPre) } \\
\hline & $x$ & $y$ & $z$ & Cluster size & $Z$ score & pSVC & $x$ & $y$ & $z$ & Cluster size & $Z$ score & pSvc \\
\hline $\begin{array}{l}\text { Heschl gyrus (Lockwood } \\
\text { et al., 1999) }\end{array}$ & 44 & -24 & 14 & 84 & 4.17 & 0.002 & & & & & & \\
\hline $\begin{array}{l}\text { Superior temporal gyrus (STG; } \\
\text { Czisch et al., 2009) }\end{array}$ & & & & & & & 68 & -44 & 18 & 49 & 3.45 & 0.017 \\
\hline
\end{tabular}

Coordinates $(x, y, z)$ are expressed in millimeter in the Montreal Neurological Institute (MNI); Z scores result from the statistical parametric analysis; $p_{\text {SVC }}$ refers to the probability of the null hypothesis (i.e., absence of activity change associated with tones in deep NREM sleep), after correction for multiple comparisons either on the whole brain volume (*) or on small volumes of interest identified in the literature (references in brackets). 


\section{A - Tone responses independent of SW phase}
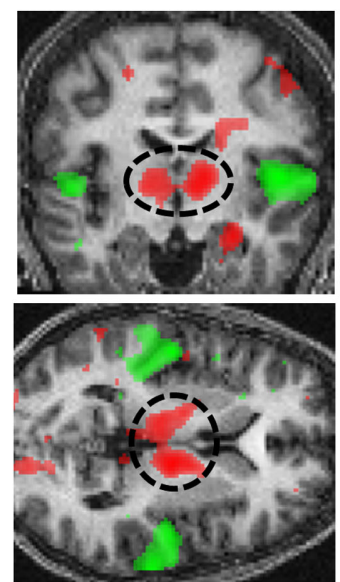

Thalamus

$-14-142$
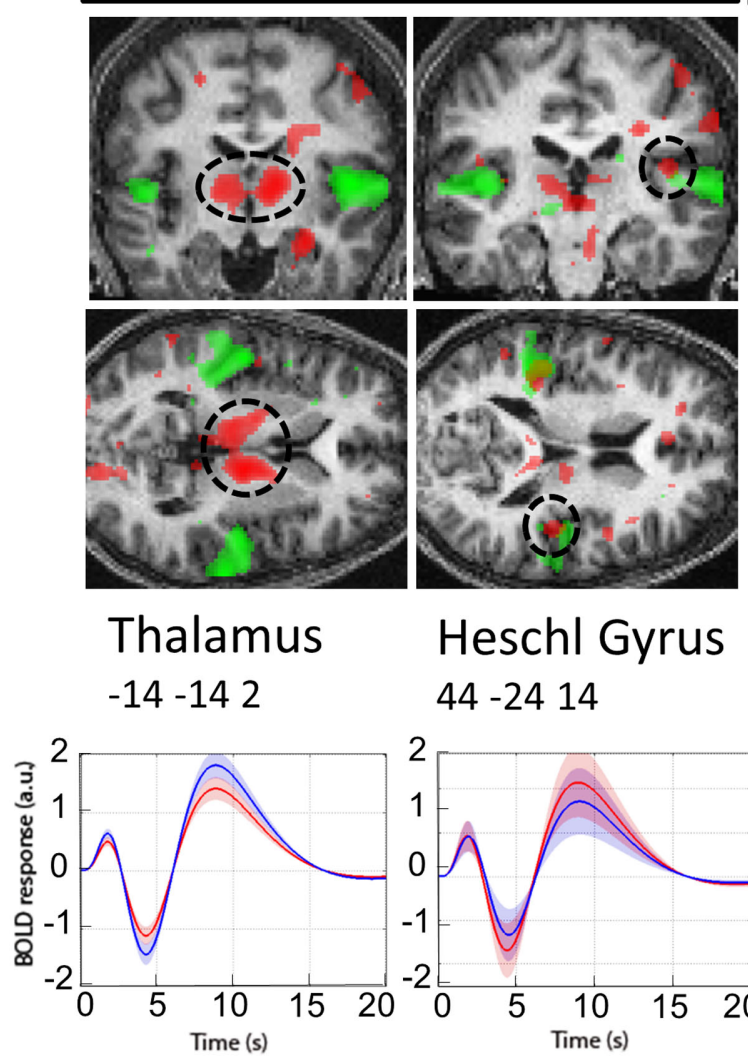

$44-2414$

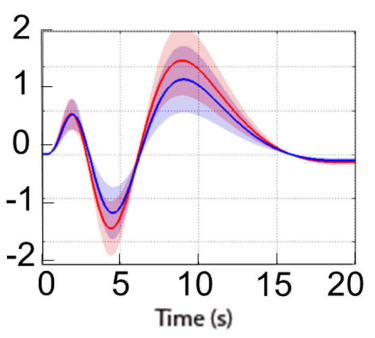

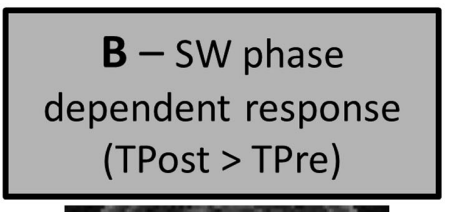
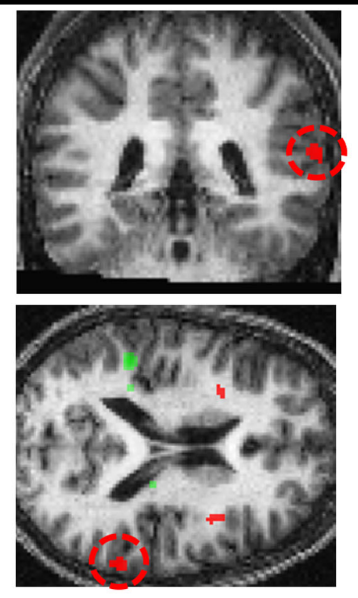

STG

\section{$68-4418$}

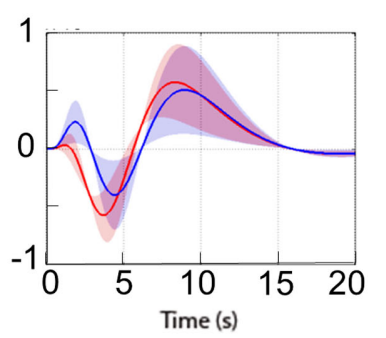

FIGURE 3 | Brain regions activated as a function of the phase of the slow oscillation in deep NREM sleep. While in the left panel (A) red color indicates that the thalamus and primary auditory cortex responds to tones across all phases of the slow oscillation, the right panel (B) depicts in red the dependence of higher auditory cortex activation (STG) on the phase of the slow-waves (SWs; TPost > TPre) during sound occurrence. Lower panels depict the fitted responses in the thalamus (left panel), the
Heschl gyrus (middle panel), and the STG (right panel) associated with sounds delivered after (TPost, blue) or before (TPre, red) the SW-peak negativity. The curves correspond to the mean and the shaded areas to the SEM. Additionally, the waking responses to tones (TW) are shown in green for illustrative purposes. Random effect results $(n=13)$ are displayed on an individual structural image (displayed at $p<0.001$, uncorrected).
Furthermore, we tested whether the amplitude of the slow oscillation during sound delivery has a modulatory effect on the subsequent differences (TPre vs. TPost) in the positive component after tone delivery. We revealed that the positive component following tone delivery is markedly enhanced if the tone is arriving at the positive going slope of the slow oscillation (TPost) in both amplitude conditions (peak amplitude small and big SW: $t_{11}=2.21$, $p<0.05$ and $t_{11}=5.91, p<0.001$, respectively; or positive area under component for small and big SW: $t_{11}=3.63, p=0.004$; $t_{11}=6.00, p<0.001$, respectively). Yet, the TPre to TPost difference for the positive component was identical for tones arriving during small or big SWs.

\section{DISCUSSION}

Our study characterizes the modulation of brain responses to auditory stimulation by spontaneous NREM sleep oscillations (sleep spindles and slow oscillations). As expected, sounds during wakefulness elicited responses in the thalamus and primary auditory cortex. These responses - although somewhat altered in size and location - persisted during NREM sleep, except during presence of light NREM spindles. Yet interestingly, responses at a higher cortical level became less consistent or even absent during spindles and the negative going phase of the (deep NREM) slow oscillation.

At the EEG level we found that ERPs elicited during NREM sleep showed a reduction in the amplitude of the N1 and an increase in the amplitude of the P2 component compared to the ERPs during wakefulness (Figure 4A) in accordance with previous studies (Campbell et al., 1992; Elton et al., 1997; Cote et al., 2000).

\section{TRANSMISSION DURING SPINDLES}

Sleep spindle results clearly indicate that brain responses and the processing of external information are for the most part absent 


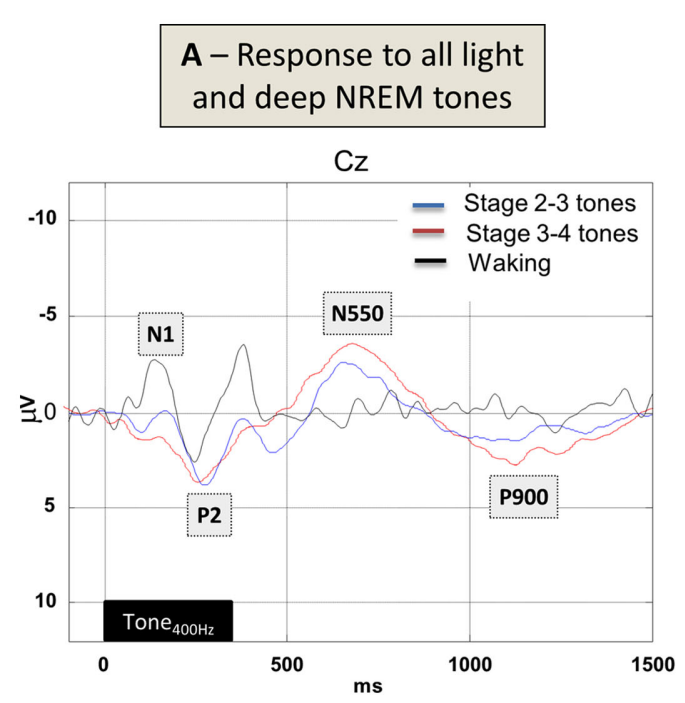

FIGURE 4 | Grand-averaged EEG brain activity in response to sounds during (A) waking and NREM sleep as well as (B) in response to the occurrence of sleep spindle events. Note the decreasing signal complexity from waking (black line), to light (red) to deep (blue) NREM sleep (left panel) in response to tones. On the right, EEG responses to

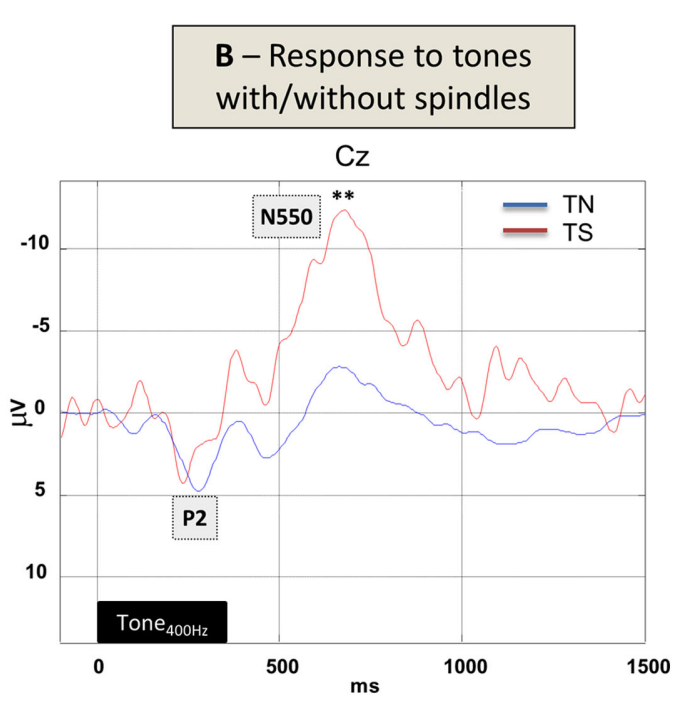

tones presented during (TS, red line) or outside spontaneously occurring stage 2-3 sleep spindles (TN, blue line) are depicted. Note the massive late negativity evoked by tones falling into sleep spindles. Data are bandpass filtered between 0.5 and $20 \mathrm{~Hz}$ and reflect the grand-average of 12 subjects.
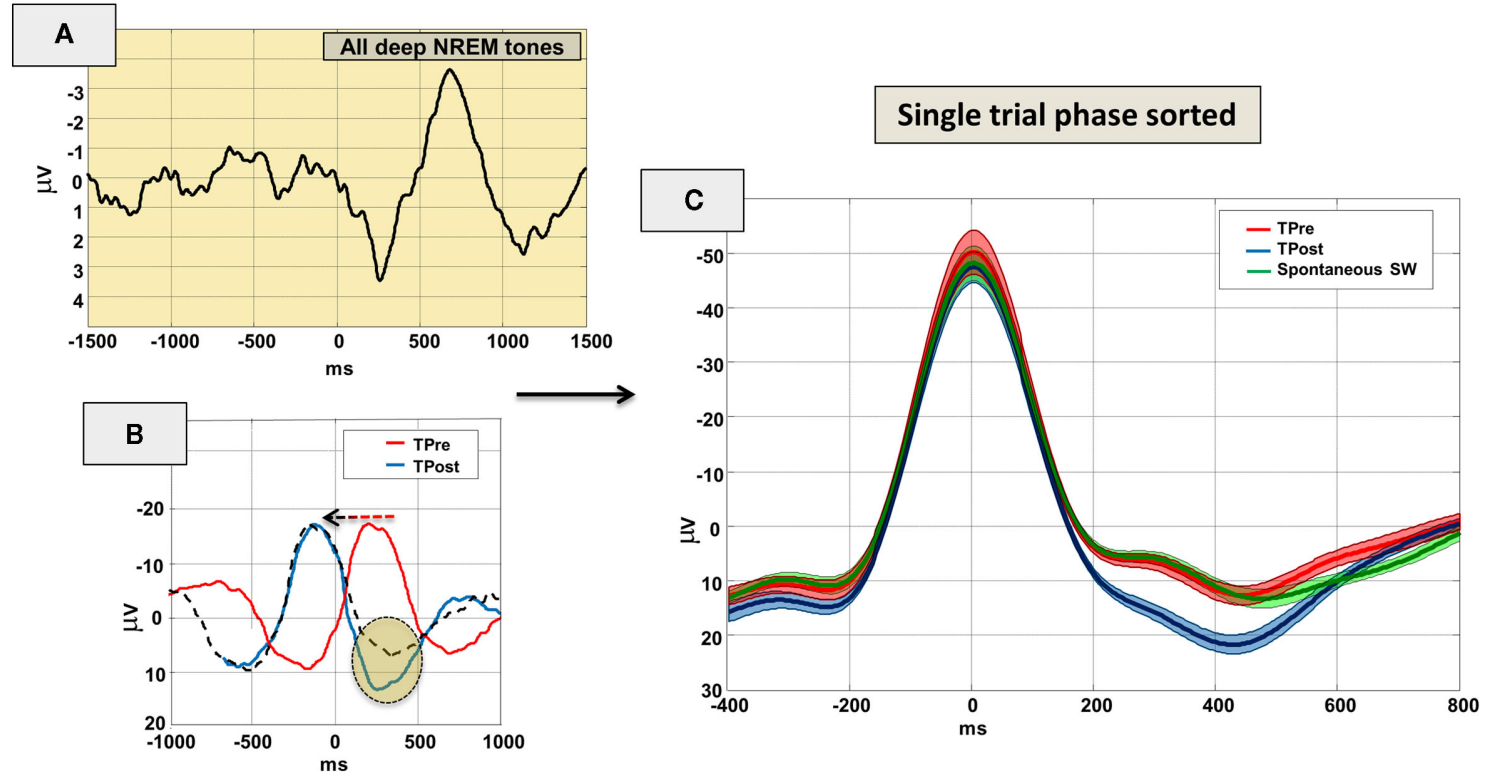

FIGURE 5 | Electroencephalography brain responses to sounds during deep NREM sleep. On the upper left (A) the evoked response to all tones during stage 3-4 is depicted. The lower left panel (B) shows the evoked response if tones are categorized according to the phase of the slow oscillation which was present during sound delivery. Note that tones occurring before the SW-peak negativity (TPre, red line) are differently processed (orange shading) than tones arriving at the upswing of the slow oscillation (TPost, blue line). The black dashed line depicts the (phase unsorted) TPre grand-average waveform which was shifted in time to the left so that their SW-peak negativity overlays with the TPost peak negativity for better comparison. Data are bandpass filtered between 0.5 and $20 \mathrm{~Hz}$ and reflect the grand-average of 12 subjects. Time 0 is the tone onset. (C) Single trial phase-sorted and re-aligned EEG brain responses to sounds during deep NREM sleep. Note that latter analysis accentuates the amplitude as no time jitter is present when averaging TPre and TPost trials according to SW-peak negativity (rather than to tones) on a trial-by-trial basis. In (C) Time 0 is therefore marking the SW-peak negativity for TPre and TPost trials. Spontaneous slow-waves are plotted for comparison in green. Data are (zero-phase lag) low-pass filtered at $4 \mathrm{~Hz}$ and error bars are overlaid at each sampling point. All potentials with negativity upwards. 
when that information is occurring during the presence of a NREM sleep spindle. Likely this is due to the fact that thalamic neurons adopt a burst firing mode during NREM sleep which prevents faithful sensory transmission of external inputs to the cortex. Presumably, exactly that functional isolation of sensory input during sleep spindles is of benefit for internal neuronal interactions subserving brain plasticity.

Contrary to Elton et al. (1997) but in line with Cote et al. (2000) we did not find an increased P2 amplitude for stimuli occurring during a spindle as compared to stimuli outside a spindle (Figure 4B). Yet, most interestingly tones presented during a spindle elicited a much stronger N550 component than tones presented outside a spindle (Figure 4B). According to Bastien and colleagues (2002) averaging responses to tones presented during sleep, which include K-complexes, produce a N550 component. In addition also Church et al. (1978) showed that auditory evoked Kcomplexes were larger when stimuli were presented during sleep spindles. It is thus possible that also the strong N550 effect seen in our data is due to the superposition of underlying K-complexes. In relation to the literature it is also interesting to note that we only find a marginally enhanced P2 component across light and deep sleep (Figure 4A); Yet we do find a strong N550 effect which is consistent with the idea that inhibition of the incoming auditory information is not occurring at an early (as reflected by P2) but later stage (in time and brain hierarchy) in accordance with our fMRI results (cf. Figure 3B).

Note that also the late negative swing of our BOLD response (at 6-8 s for spindles, and at $4-6 \mathrm{~s}$ for SWs) could be related to underlying K-complexes on some trials. Specifically it is interesting to note that Czisch et al. (2009) using an acoustic oddball paradigm reported a prominent negative BOLD response for (rare) tones, yet no wake-like activation of the auditory cortex. In their data only rare tones followed by an evoked K-complex, were associated with a "wake-like activation of task-related areas in the temporal cortex" in accordance with data from Dang-Vu et al. (2011). Our current fMRI results showing auditory cortex activation (in the absence of sleep spindles and across SW phases) as well as a late negative BOLD swing might thus be interpreted as combination of these two effects. However, note that in our data a K-complex like N550 effect appears only when averaging EEG across all SW phases (cf. Figure 4A, red line) or tones in the presence of sleep spindles (cf. Figure 4B, red line), but not in response to tones just before or after the SW-peak negativity (cf. Figure 5B).

Yet, the exact anatomical stage at which the transfer of sensory information to auditory thalamo-cortical pathways is hindered remains uncertain. It appears that no clear-cut change in neural activity takes place at any of the early auditory relay structures during NREM sleep (Velluti, 2008), but prethalamic modifications in transmission were reported in the somato-sensory system during NREM sleep, although not specifically in relation to spindles (Rosanova and Timofeev, 2005). On the other hand, thalamic neurons are likely to hinder the faithful transmission of sensory input during spindles, because the burst firing mode that they adopt during NREM sleep, and especially during spindles, distorts the transmission of sensory inputs to the cortex in a non-linear fashion (McCormick and Feeser, 1990; Sherman and Guillery, 2002). Finally, also note that a strong recruitment of inhibitory interneurons was recently described during sleep spindles (Peyrache et al., 2011). This phenomenon might add to the absence of cortical responses to auditory stimuli during spindles as observed in our previous study and reiterated herein.

\section{PHASE DEPENDENCE OF BRAIN RESPONSES WITH RESPECT TO THE SLOW OSCILLATION}

While the phase of the slow oscillation does not appear to alter brain responses in primary sensory cortex (cf. Figure 3A), it does modulate responses at higher cortical levels as shown in superior temporal gyrus (cf. Figure 3B; for additional areas also see Table S1 in Supplementary Material). On the one hand, the results are consistent with the hypothesis that brain responses during deep NREM sleep vary as a function of the fluctuating state of thalamocortical circuits (Massimini et al., 2003; Rosanova and Timofeev, 2005). In accordance with Massimini and colleagues the brain appears most receptive to the environment at the negative-topositive going slope of the slow oscillation. Yet, a direct comparison to our data is difficult as stimulation was done in another sensory modality (somatosensory vs. auditory) and EEG components of interest were markedly earlier. Last but not least we compared pre- vs. post-SW-peak phases (300 ms bins each) whereas Massimini and colleagues used smaller and differently grouped time windows (e.g., $\pm 50 \mathrm{~ms}$ directly around the SW-peak negativity). On the other hand, the current data suggest that the slow oscillation does only modulate responses in higher associative cortices. In keeping with this observation, the slow oscillation was also found to be more associated with a pronounced modulation of neuronal firing in associative than in primary cortices at a cellular level (Steriade and McCarley, 2005). This response pattern also concords with the breakdown of local functional connectivity reported during NREM sleep (Massimini et al., 2005) and is reminiscent of auditory responses recorded in unconscious patients in whom primary auditory cortices still respond whereas higher association cortices do not (Laureys et al., 2000; Boly et al., 2004). The results suggest that the residual cortical processing during NREM sleep is insufficient for ignition of processes thought to be necessary for conscious awareness but might very well allow to trigger awakening responses to salient and personally important stimuli.

The analysis of EEG data simultaneously recorded during fMRI sessions further dissect the reciprocal interactions between spontaneous brain activity and responses to external stimuli. Importantly, EEG data allow us to probe how the intrinsic state of neural responsiveness (i.e., SW phase) influences responses to external stimuli. They allow us to go one step closer to the actual neural events subtending sound processing during NREM sleep although the exact phase relation between the scalp-recorded human sleep EEG and the underlying intracellular dynamics can only be extrapolated from animal experiments (Massimini et al., 2003; Vyazovskiy et al., 2011).

Tones delivered after the peak negativity (i.e., possibly during the ON state) probably contribute to further synchronize neural firing (cf. Figure 5), already ongoing due to the ON state. Tones delivered before SW-peak negativity (supposedly at the end of the OFF state) potentially initiate the cascade of events that lead to the $\mathrm{ON}$ state. The smaller amplitude of the second positive evoked component might suggest that the neural recruitment induced by sounds in the negative going slope (TPre) is smaller as compared to the - probably synchronized - TPost response during the positive 
going phase. On the other hand one could argue that the auditory evoked potential during deep NREM sleep - presumably reflecting a K-complex related N550 around 650 ms (cf. Figure 5A) - “attenuates" the late positive component (also around $650 \mathrm{~ms}$ ) of the tones arriving before SW-peak negativity (Figure 5B). Yet, the reconstruction of a spontaneous SW without tone delivery (cf. Figure 5C, green line) clearly favors the earlier interpretation. In either case the EEG data reveal a clear phase-dependent modulation of the auditory ERP response post-event which is not present before tone delivery (Figure 5C).

Future studies should elaborate on the reported findings and investigate phase-dependent effects of smaller temporal windows (100 ms or less) as well as auditory stimuli of varying complexity.

\section{CONCLUSION}

Present evidence extends previous findings and suggests that SWS is not a static phenomenon as mutually assumed by many earlier studies (Perrin et al., 1999; Portas et al., 2000; Bastuji et al., 2002; Campbell et al., 2005). Altogether, brain responses during NREM sleep appear to be non-stationary and highly dependent upon spontaneous brain activity such as prominent sleep spindles or the phase of the slow oscillation. The exact temporal window when a stimulus arrives might thus not only determine the fate of that very material during waking but likewise all stages of NREM sleep.

\section{AUTHOR CONTRIBUTIONS}

Manuel Schabus, Thien Thanh Dang-Vu, and Pierre Maquet contributed at all stages of the manuscript. Manuel Schabus and

\section{REFERENCES}

Allen, P. J., Josephs, O., and Turner, R. (2000). A method for removing imaging artifact from continuous EEG recorded during functional MRI. Neuroimage 12, 230-239.

Bastien, C. H., Crowley, K. E., and Colrain, I. M. (2002). Evoked potential components unique to non-REM sleep: relationship to evoked $\mathrm{K}$ complexes and vertex sharp waves. Int. J. Psychophysiol. 46, 257-274.

Bastuji, H., and Garcia-Larrea, L. (1999). Evoked potentials as a tool for the investigation of human sleep. Sleep Med. Rev. 3, 23-45.

Bastuji, H., Perrin, F., and Garcia-Larrea, L. (2002). Semantic analysis of auditory input during sleep: studies with event related potentials. Int. J. Psychophysiol. 46, 243-255.

Boly, M., Balteau, E., Schnakers, C., Degueldre, C., Moonen, G., Luxen, A., Phillips, C., Peigneux, P., Maquet, P., and Laureys, S. (2007). Baseline brain activity fluctuations predict somatosensory perception in humans. Proc. Natl. Acad. Sci. U.S.A. 104, 12187-12192.

Boly, M., Faymonville, M. E., Peigneux, P., Lambermont, B., Damas, P., Del Fiore, G., Degueldre, C., Franck,
G., Luxen, A., Lamy, M., Moonen, G., Maquet, P., and Laureys, S. (2004). Auditory processing in severely brain injured patients: differences between the minimally conscious state and the persistent vegetative state. Arch. Neurol. 61, 233-238.

Buysse, D. J., Reynolds, C. F. III, Monk, T. H., Berman, S. R., and Kupfer, D. J. (1989). The Pittsburgh Sleep Quality Index: a new instrument for psychiatric practice and research. Psychiatry Res. 28, 193-213.

Campbell, K., Bell, I., and Bastien, C. (1992). "Evoked potential measures of information processing during natural sleep," in Sleep, Arousal, and Performance, eds R. J. Broughton and R. D. Ogilvie (Boston: Birkhauser Press), 88-116.

Campbell, K., Michaud, D. S., Keith, S. E., Muller-Gass, A., and Wiebe, S. (2005). Event-related potential measures of the disruptive effects of trains of auditory stimuli during waking and sleeping states. J. Sleep Res. 14, 347-357.

Campbell, K. B., and Colrain, I. M. (2002). Event-related potential measures of the inhibition of information processing: II. The sleep

Pierre Maquet wrote up the manuscript. These and all of the following authors (Annabelle Darsaud, Christina Schmidt, Gilles Vandewalle, Mélanie Boly, Martin Desseilles, Steffen Gais) were involved in data recording, discussed the results or commented on the manuscript. Dominik Philip Johannes Heib, Christian Degueldre, Christophe Phillips, and Evelyne Balteau added important technical expertise.

\section{ACKNOWLEDGMENTS}

This study was supported by the Belgian Fonds National de la Recherche Scientifique (F.N.R.S.), the Fondation Médicale Reine Elisabeth, the Research Fund of ULg, and PAI/IAP Interuniversity Pole of Attraction P5/04. Geneviève Albouy is supported by a PhD grant from the French "Ministère de la Recherche." Gilles Vandewalle, Evelyne Balteau, Mélanie Boly, Thien Thanh Dang-Vu, Martin Desseilles, Christophe Phillips, Annabelle Darsaud, and Pierre Maquet are supported by F.N.R.S. Thien Thanh Dang-Vu is also supported by the Fonds Léon Frédéricq (Belgium), the Belgian College of Neuropsychopharmacology and Biological Psychiatry, and the Canadian Institutes of Health Research. Steffen Gais is supported by an Emmy Noether fellowship from the German Research Foundation (DFG). Manuel Schabus is supported by an Erwin Schrödinger fellowship of the Austrian Science Fund (FWF; J2470-B02).

\section{SUPPLEMENTARY MATERIAL}

The Supplementary Material for this article can be found online at http://www.frontiersin.org/sleep_and_chronobiology/abstract/ 18937

onset period. Int. J. Psychophysiol.46, 197-214.

Church, M. W., Johnson, L. C., and Seales, D. M. (1978). Evoked K-complexes and cardiovascular responses to spindle-synchronous and spindle-asynchronous stimulus clicks during NREM sleep. Electroencephalogr. Clin. Neurophysiol. 45, 443-453.

Colrain, I. M., and Campbell, K. B. (2007). The use of evoked potentials in sleep research. Sleep Med. Rev. 11, 277-293.

Contreras, D., Timofeev, I., and Steriade, M. (1996). Mechanisms of long lasting hyperpolarizations underlying slow sleep oscillations in cat corticothalamic networks. J. Physiol. (Lond.) 494, 251-264.

Cote, K. A., Epps, T. M., and Campbell, K. B. (2000). The role of the spindle in human information processing of high-intensity stimuli during sleep. J. Sleep Res. 9, 19-26.

Crowley, K. E., and Colrain, I. M. (2004). A review of the evidence for $\mathrm{P} 2$ being an independent component process: age, sleep and modality. Clin. Neurophysiol. 115, 732-744.

Czisch, M., Wehrle, R., Kaufmann, C., Wetter, T. C., Holsboer, F.,
Pollmacher, T., and Auer, D. P. (2004). sFunctional MRI during sleep: BOLD signal decreases and their electrophysiological correlates. Eur. J. Neurosci. 20, 566-574.

Czisch, M., Wehrle, R., Stiegler, A., Peters, H., Andrade, K., Holsboer, F., and Samann, P. G. (2009). Acoustic oddball during NREM sleep: a combined EEG/fMRI study. PLoS ONE 4, e6749. doi:10.1371/journal.pone.0006749

Czisch, M., Wetter, T. C., Kaufmann, C., Pollmacher, T., Holsboer, F., and Auer, D. P. (2002) Altered processing of acoustic stimuli during sleep: reduced auditory activation and visual deactivation detected by a combined fMRI/EEG study. Neuroimage 16, 251-258.

Dang-Vu, T. T., Bonjean, M., Schabus, M., Boly, M., Darsaud, A., Desseilles, M., Degueldre, C., Balteau, E., Phillips, C., Luxen, A., Sejnowski, T. J., and Maquet, P. (2011). Interplay between spontaneous and induced brain activity during human non-rapid eye movement sleep. Proc. Natl. Acad. Sci. U.S.A. 108, 15438-15443. 
Dang-Vu, T. T., Mckinney, S. M., Buxton, O. M., Solet, J. M., and Ellenbogen, J. M. (2010). Spontaneous brain rhythms predict sleep stability in the face of noise. Curr. Biol. 20, R626-R627.

Dang-Vu, T. T., Schabus, M., Desseilles, M., Albouy, G., Boly, M., Darsaud, A., Gais, S., Rauchs, G., Sterpenich, V., Vandewalle, G., Carrier, J., Moonen, G., Balteau, E., Degueldre, C., Luxen, A., Phillips, C., and Maquet, P. (2008). Spontaneous neural activity during human slow wave sleep. Proc. Natl. Acad. Sci. U.S.A. 105, 15160-15165.

de Lugt, D. R., Loewy, D. H., and Campbell, K. B. (1996). The effect of sleep onset on event related potentials with rapid rates of stimulus presentation. Electroencephalogr. Clin. Neurophysiol. 98, 484-492.

Elton, M., Winter, O., Heslenfeld, D., Loewy, D., Campbell, K., and Kok, A. (1997). Event-related potentials to tones in the absence and presence of sleep spindles. J. Sleep Res. 6, 78-83.

Gaab, N., Gaser, C., Zaehle, T., Jancke, L., and Schlaug, G. (2003). Functional anatomy of pitch memory - an fMRI study with sparse temporal sampling. Neuroimage 19, 1417-1426.

Hanslmayr, S., Aslan, A., Staudigl, T., Klimesch, W., Herrmann, C. S., and Bauml, K. H. (2007). Prestimulus oscillations predict visual perception performance between and within subjects. Neuroimage 37, 1465-1473.

Hesselmann, G., Kell, C. A., Eger, E., and Kleinschmidt, A. (2008a). Spontaneous local variations in ongoing neural activity bias perceptual decisions. Proc. Natl. Acad. Sci. U.S.A. 105, 10984-10989.

Hesselmann, G., Kell, C. A., and Kleinschmidt, A. (2008b). Ongoing activity fluctuations in hMT+ bias the perception of coherent visual motion. J. Neurosci. 28, 14481-14485.

Holcomb, H. H., Medoff, D. R., Caudill, P. J., Zhao, Z., Lahti, A. C., Dannals, R. F., and Tamminga, C. A. (1998). Cerebral blood flow relationships associated with a difficult tone recognition task in trained normal volunteers. Cereb. Cortex 8 , 534-542.

Horne, J. A., and Ostberg, O. (1976). A self-assessment questionnaire to determine morningness-eveningness in human circadian rhythms. Int. J. Chronobiol. 4, 97-110.

Johns, M. W. (1991). A new method for measuring daytime sleepiness: the Epworth sleepiness scale. Sleep 14, 540-545.

Laureys, S., Faymonville, M. E., Degueldre, C., Fiore, G. D., Damas, P., Lambermont, B., Janssens, N., Aerts, J., Franck, G., Luxen, A., Moonen, G., Lamy, M., and Maquet, P. (2000). Auditory processing in the vegetative state. Brain 123(Pt 8), 1589-1601.

Lockwood, A. H., Salvi, R. J., Coad, M. L., Arnold, S. A., Wack, D. S. Murphy, B. W., and Burkard, R. F. (1999). The functional anatomy of the normal human auditory system: responses to 0.5 and $4.0 \mathrm{kHz}$ tones at varied intensities. Cereb. Cortex 9 , 65-76.

Massimini, M., and Amzica, F. (2001). Extracellular calcium fluctuations and intracellular potentials in the cortex during the slow sleep oscillation. J. Neurophysiol. 85, 1346-1350.

Massimini, M., Ferrarelli, F., Huber, R., Esser, S. K., Singh, H., and Tononi, G. (2005). Breakdown of cortical effective connectivity during sleep. Science 309, 2228-2232.

Massimini, M., Rosanova, M., and Mariotti, M. (2003). EEG slow (approximately $1 \mathrm{~Hz}$ ) waves are associated with nonstationarity of thalamocortical sensory processing in the sleeping human. J. Neurophysiol. 89, 1205-1213.

McCormick, D. A., and Feeser, H. R. (1990). Functional implications of burst firing and single spike activity in lateral geniculate relay neurons. Neuroscience 39, 103-113.

Mölle, M., Marshall, L., Gais, S., and Born, J. (2002). Grouping of spindle activity during slow oscillations in human non-rapid eye movement sleep. J. Neurosci. 22, 10941-10947.

Näätänen, R., Teder, W., Alho, K., and Lavikainen, J. (1992). Auditory attention and selective input modulation: a topographical ERP study. Neuroreport 3, 493-496.

Novitski, N., Alho, K., Korzyukov, O., Carlson, S., Martinkauppi, S., Escera, C., Rinne, T., Aronen, H. J., and Naatanen, R. (2001). Effects of acoustic gradient noise from functional magnetic resonance imaging on auditory processing as reflected by event-related brain potentials. Neuroimage 14, 244-251.

Palva, S., Linkenkaer-Hansen, K., Naatanen, R., and Palva, J. M. (2005). Early neural correlates of conscious somatosensory perception. J. Neurosci. 25, 5248-5258.

Perrin, F., Garcia-Larrea, L., Mauguiere, F., and Bastuji, H. (1999). A differential brain response to the subject's own name persists during sleep. Clin. Neurophysiol. 110 2153-2164.

Peyrache, A., Battaglia, F. P., and Destexhe, A. (2011). Inhibition recruitment in prefrontal cortex during sleep spindles and gating of hippocampal inputs. Proc. Natl. Acad. Sci. U.S.A. 108, 17207-17212.

Portas, C. M., Krakow, K., Allen, P. Josephs, O., Armony, J. L., and Frith, C. D. (2000). Auditory processing across the sleep-wake cycle: simultaneous EEG and fMRI monitoring in humans. Neuron 28, 991-999.

Raichle, M. E. (2006). Neuroscience. The brain's dark energy. Science 314, 1249-1250.

Raichle, M. E., and Mintun, M. A. (2006). Brain work and brain imaging. Annu. Rev. Neurosci. 29, 449-476.

Rechtschaffen, A., and Kales, A. A. (1968). A Manual of Standardized Terminology, Techniques and Scoring System for Sleep Stages of Human Sujects. Bethesda: US Department of Health, Education, and Welfare Public Health Service - NIH/ NIND.

Rosanova, M., and Timofeev, I. (2005). Neuronal mechanisms mediating the variability of somatosensory evoked potentials during sleep oscillations in cats. J. Physiol. (Lond.) 562, 569-582.

Schabus, M., Dang-Vu, T. T., Albouy, G., Balteau, E., Boly, M., Carrier, J., Darsaud, A., Degueldre, C., Desseilles, M., Gais, S., Phillips, C., Rauchs, G.,Schnakers, C., Sterpenich, V., Vandewalle, G., Luxen, A., and Maquet, P. (2007). Hemodynamic cerebral correlates of sleep spindles during human non-rapid eye movement sleep. Proc. Natl. Acad. Sci. U.S.A. 104, 13164-13169.

Sherman, S. M., and Guillery, R. W. (2002). The role of the thalamus in the flow of information to the cortex.
Philos. Trans. R. Soc. Lond. B Biol. Sci. 357, 1695-1708.

Srivastava, G., Crottaz-Herbette, S., Lau, K. M., Glover, G. H., and Menon, V. (2005). ICA-based procedures for removing ballistocardiogram artifacts from EEG data acquired in the MRI scanner. Neuroimage 24, 50-60.

Steriade, M. (1991). "Alertness, quiet sleep, dreaming," in Cerebral Cortex, Vol. 9, Normal and Altered States of Function, eds A. Peters and E. J. Jones (New York: Plenum Press), 279-357.

Steriade, M., and McCarley, R. W. (2005). Brain Control of Wakefulness and Sleep. New York: Springer.

Velluti, R. A. (2008). The Auditory System In Sleep. San Diego: Academic Press.

Vyazovskiy, V. V., Cirelli, C., and Tononi, G. (2011). Electrophysiological correlates of sleep homeostasis in freely behaving rats. Prog. Brain Res. 193, 17-38.

Conflict of Interest Statement: The authors declare that the research was conducted in the absence of any commercial or financial relationships that could be construed as a potential conflict of interest.

Received: 14 November 2011; paper pending published: 15 December 2011; accepted: 02 March 2012; published online: 05 April 2012.

Citation: Schabus M, Dang-Vu TT, Heib DPJ, Boly M, Desseilles M, Vandewalle $G$, Schmidt C, Albouy G, Darsaud A, Gais $S$, Degueldre C, Balteau E, Phillips C, Luxen A and Maquet $P$ (2012) The fate of incoming stimuli during NREM sleep is determined by spindles and the phase of the slow oscillation. Front. Neur. 3:40. doi: 10.3389/fneur.2012.00040

This article was submitted to Frontiers in Sleep and Chronobiology, a specialty of Frontiers in Neurology.

Copyright (C) 2012 Schabus, Dang$V u$, Heib, Boly, Desseilles, Vandewalle, Schmidt, Albouy, Darsaud, Gais, Degueldre, Balteau, Phillips, Luxen and Maquet. This is an open-access article distributed under the terms of the Creative Commons Attribution Non Commercial License, which permits noncommercial use, distribution, and reproduction in other forums, provided the original authors and source are credited. 